\title{
ACOGIMIENTO RESIDENCIAL Y PRODUCCIÓN DE SUBJETIVIDADES: IDENTIDADES Y TRAYECTORIAS CIUDADANAS DE LOS NIÑOS Y NIÑAS EN PROTECCIÓN
}

\author{
Child residential care and the production of subjectivity: \\ children's indentities and citizenship trajectories
}

JULIA RAMIRO

\section{Resumen}

El artículo analiza los procesos identitarios de los niños/as y adolescentes en acogimiento residencial, relacionados con la construcción de sus trayectorias ciudadanas. Siguiendo el método biográfico y realizando entrevistas abiertas en profundidad, se exploran tanto el impacto de los valores institucionales como la incidencia de las normas y rutinas residenciales en la producción de sus subjetividades. Desde el enfoque de la ciudadanía vivida, se muestra cómo los niños y niñas acogidos representan, reelaboran e interactúan con el sistema de normas y valores de la protección a la infancia. El análisis del material empírico pone de manifiesto las tensiones existentes en los relatos de los niños/as entrevistados en torno a sus identidades y pertenencias y su visión particular sobre el ser ciudadano. Ambas cuestiones deben ser vistas como consecuencia del impacto del marco simbólico de la protección y el producto (aunque reelaborado) del conjunto de prácticas cotidianas en los recursos de acogimiento residencial.

Palabras clave. Niños/as en protección, acogimiento residencial, Identidades y pertenencias, trayectorias ciudadanas.

\begin{abstract}
The article analyzes the identity processes of children in residential care related to their construction of citizenship trajectories. Using a biographical method and in-depth interviews, this study explores the impact of institutional values and the influence of rules and routines on the children's production of subjectivity. Taking into account the citizenship from below approach, it shows how children living in care picture and interact with the regulations and values of the Child Protection System. The analysis of the empirical material illustrates relevant tensions involved in the children's narratives about their identities and belongings and their visions about being a citizen. Both might be seen as a result of the impact of the symbolic protection framework and the consequence of the set of daily practices in residential care institutions.
\end{abstract}

Keywords. Children living in care, Residential care, Identities and belongings, Citizenship trajectories.

Recibido: 03/06/2015

Aceptado: 10/07/2015

Publicado: 03/12/2015

1. Universidad Nacional de Educación a Distancia. Facultad de Derecho. Dpto. Servicios Sociales y Fundamentos Histórico-jurídicos. C/ Obispo Trejo, 2 CP: 28040, Madrid. jramiro@der.uned.es 


\section{Introducción}

En la actualidad, el acogimiento residencial se encuentra en el epicentro de los debates sociales sobre la protección a la infancia. Frente a otros países europeos y pese al reciente esfuerzo desinstitucionalizador, iniciado a tenor de la Comisión Especial del Senado para estudiar la problemática de la adopción nacional y los temas afines relacionados con ella, como acogimiento, desamparo e institucionalización (2008) y del Anteproyecto de Ley Orgánica del Sistema de protección a la Infancia y a la Adolescencia (2011), España sigue registrando altas tasas de institucionalización. Aunque no existen datos claros y actualizados, sólo en el año 2013 se adoptaron un total de 35.045 medidas de acogimiento y, de éstas, el 38,2\% correspondieron al acogimiento residencial, con una estimación de 13.041 niños/as y adolescentes derivados a los diferentes recursos de protección a lo largo del territorio español. Estos datos reflejan, por un lado, la necesidad de profundizar en la comprensión del papel actual del acogimiento residencial y, por otro, de conocer cómo incide en la vida de los niños/as y adolescentes acogidos.

En este contexto, la integración cívica y social de los niños/as institucionalizados se ha planteado como uno de los principales objetos de preocupación social desde la reforma jurídica de protección a la infancia de 1987, con la Ley 21/1987 de 11 de noviembre, por la que se modifican determinados artículos del Código Civil y de la Ley de Enjuiciamiento Civil en materia de adopción. Desde entonces, la idea de normalización opera como principio rector en el diseño y organización de los recursos residenciales para la infancia en protección. Así, recientemente, el estudio Estándares de calidad en acogimiento residencialEQUAR (2012) $)^{2}$ señalaba los estudios y formación, la normalización e integración, el desarrollo y autonomía y el uso de las consecuencias educativas como predictores de integración social y bienestar de los niños/as acogidos. La enunciación de dichas condiciones, como parte constitutiva de la calidad en el acogimiento residencial, remite a las funciones históricas de la protección a la infancia: regulación, control y ordenamiento de las ciudadanías (Ramiro, 2015). Por ello, se entiende que: «El trabajo de atención residencial debe partir de considerar al niño como un sujeto en desarrollo y, por tanto, con una especial necesidad de ser estimulado, guiado y protegido hacia unos objetivos de madurez y socialización que en muchos casos tendrán que contemplar la transición a la vida adulta e independiente» (EQUAL, 2012: 68).

2. Investigación realizada por la Asociación NIERU, con el apoyo del Grupo de Investigación en Familia e Infancia de la Universidad de Oviedo, coordinada por FAPMI y financiada por el Ministerio de Sanidad, Servicios Sociales e Igualdad. 
La asociación del concepto de ciudadanía con la adultez es característica de un modelo clásico que tradicionalmente, desde el plano legal y formal, ha excluido a los niños por motivos de dependencia emocional e inmadurez psicológica. Sin embargo, esta idea ha sido ampliamente contestada desde las ciencias sociales (Cockburn, 1998; Cohen, 2005; Lister, 2007; Moosa-Mitha, 2005; Roche, 1999), alegando que una sociedad comprometida e inclusiva con los niños/as ha de partir de una concepción de la ciudadanía más amplia. De hecho, la ciudadanía es, para todos, más que un conjunto de derechos reconocidos política y legalmente. Se trata también de un proceso relacional que incluye dimensiones sustantivas tales como las responsabilidades, la participación, las identidades y pertenencias (Lister, 2007).

El presente trabajo centra su interés en la última dimensión señalada y en cómo ésta incide en la construcción de la ciudadanía entre los niños/as y jóvenes institucionalizados. Por tanto, el artículo explora los procesos identitarios de los niños/as y adolescentes protegidos en el marco de los recursos residenciales, teniendo en cuenta que éstos se erigen como estructuras de normalización y control social.

Cabe destacar que, en el marco de la intervención social y del trabajo social, con este trabajo, se pretende devolver el protagonismo a los niños/as y adolescentes en protección pues, si bien la imagen dominante sobre ellos se fundamenta en su «inherente» vulnerabilidad y/o potencial conflictividad, poco se conoce acerca de las experiencias de estos niños y niñas, narradas por ellos mismos, y sobre cómo responden a los valores institucionales de la protección a la infancia.

\section{Metodología}

A nivel metodológico, se han seleccionado el método biográfico y las entrevistas abiertas en profundidad como los instrumentos más adecuados para recoger las experiencias vitales de los niños/as y adolescentes protegidos durante su trayectoria por el sistema de protección. Se trata de conocer cómo los niños y niñas experimentan y responden a las conceptualizaciones institucionales que inciden en la construcción de sus identidades y de ciudadanía.

Siguiendo el enfoque del construccionismo social (Berger y Luckmann, 1967; Mead, 1934), las identidades son entendidas como interacciones complejas en continua renegociación, a través de los intercambios lingüísticos y las acciones sociales; por lo que la consideración del marco simbólico y normativo en el que se desenvuelven resulta crucial para su análisis. Así mismo, el control se propone como un proceso social imbricado en las distintas actividades propuestas en los recursos de acogimiento residencial: tareas, 
actividades de ocio y tiempo libre, posesiones, relaciones sociales, estructuración del tiempo, movilidad y privacidad (Millham, Bullock y Cherret, 1975) a modo de instituciones totales (Goffman, 1961).

En la medida en que identidades y ciudadanía son propuestas como procesos dinámicos y reinterpretativos, se hace necesario enfocar el estudio desde la perspectiva de la ciudadanía vivida (James, 2011; Liebel, 2008; Lister, 2007; Spyrou, 2008) pues, se parte de la consideración de los niños/as y adolescentes como sujetos y agentes de su propio proceso de integración social y cívica ${ }^{3}$. Postura epistemológica que, por otra parte, facilita el acceso a las representaciones, actitudes y relaciones que intervienen en la construcción de las identidades y ciudadanía de estos niños, niñas y adolescentes.

El material analizado se compone de nueve entrevistas biográficas en profundidad realizadas a jóvenes que ya han salido del sistema de protección (chicos y chicas entre 17 y 23 años), documentos personales (cuarenta y una cartas de niños/as y adolescentes acogidos en recursos de protección, recopiladas entre 2008 y 2010) y un caso/expediente de doble tutela (2012).

Para la selección de la población entrevistada se han tenido en cuenta las variables institucionales y existenciales (Ibáñez, 2003). Del cruce de ambas se obtienen los interlocutores, cubriendo así la variabilidad existente entre los recursos de protección (tipo de recurso e ideología ${ }^{4}$ ) y entre los perfiles sociodemográficos (sexo y edad) e institucionales (motivos de la protección y régimen de la misma) de los jóvenes entrevistados.

Así mismo, el campo se desarrolla en la Comunidad de Madrid, por cuanto puede ser considerada, junto con Cataluña, como una de las autonomías pioneras en incorporar las recomendaciones establecidas por la UNCRC de 1989 sobre los derechos de los niños y niñas y su ciudadanía (activa), a través de Ley 6/1995, de 28 de marzo, de Garantías de los Derechos de la Infancia y la Adolescencia y el Decreto 88/98 de 21 de Mayo de 1998: Estatuto de las Residencias de Atención a la Infancia y Adolescencia, entre otros.

3. A fin al concepto de reproducción interpretativa acunado por Corsaro (1997: 18-19) desde el que se pone de manifiesto la idea de que los niños y niñas no se limitan simplemente a interiorizar la sociedad y la cultura, si no que contribuyen activamente a dicha cultura y al cambio de la misma. En el ámbito nacional, se puede consultar la obra de Gaitán (2014) sobre los derechos de los niños en el trabajo social.

4. Se tiene en cuenta el tipo de recurso (Moss, 1975) en relación al tamaño del mismo (residencia, hogar, piso, etc.) y la ideología referida a sistemas culturales más o menos cerrados (Tizard et al., 1975): laica y/o religiosa. 
Tabla 1. Interlocutores entrevistados

\begin{tabular}{|c|c|c|l|l|}
\hline $\begin{array}{c}\text { Inter- } \\
\text { locutor }\end{array}$ & Sexo & $\begin{array}{c}\text { Edad } \\
\text { (años) }\end{array}$ & \multicolumn{1}{|c|}{$\begin{array}{c}\text { Medida y motivos de la } \\
\text { protección }\end{array}$} & \multicolumn{1}{|c|}{ Recurso } \\
\hline I1 & Mujer & 24 & $\begin{array}{l}\text { Guarda } \\
\text { Trastorno de la conducta }\end{array}$ & Centro terapéutico \\
\hline I2 & Hombre & 21 & $\begin{array}{l}\text { Guarda } \\
\text { Problemas de } \\
\text { comportamiento }\end{array}$ & $\begin{array}{l}\text { Residencia infantil pública y } \\
\text { laica y centro terapéutico }\end{array}$ \\
\hline I3 & Mujer & 21 & $\begin{array}{l}\text { Tutela } \\
\text { Abusos sexuales }\end{array}$ & $\begin{array}{l}\text { Residencia infantil privada } \\
\text { laica. }\end{array}$ \\
\hline I4 & Hombre & 19 & $\begin{array}{l}\text { Tutela } \\
\text { Abandono }\end{array}$ & $\begin{array}{l}\text { Residencia infantil privada y } \\
\text { laica y centro terapéutico }\end{array}$ \\
\hline I5 & Mujer & 20 & $\begin{array}{l}\text { Tutela } \\
\text { Negligencia y abandono }\end{array}$ & $\begin{array}{l}\text { Residencia infantil privada y } \\
\text { religiosa. }\end{array}$ \\
\hline I7 & Mujer & 18 & $\begin{array}{l}\text { Guarda y posterior tutela. } \\
\text { Pobreza }\end{array}$ & $\begin{array}{l}\text { Residencia de primera } \\
\text { acogida, residencia infantil } \\
\text { pública y laica y centro } \\
\text { terapéutico. }\end{array}$ \\
\hline I8 & Hombre & 20 & $\begin{array}{l}\text { Tutela } \\
\text { Drogodependencia parental }\end{array}$ & $\begin{array}{l}\text { Residencia infantil privada } \\
\text { laica. }\end{array}$ \\
\hline Mujer & 21 & $\begin{array}{l}\text { Puarda } \\
\text { Trastornos emocionales }\end{array}$ & $\begin{array}{l}\text { Residencia de primera } \\
\text { acogida y residencia infantil } \\
\text { privada y laica. }\end{array}$ \\
\hline
\end{tabular}

\section{Protección y acogimiento residencial en la producción de subjetividades}

Los recursos de protección, como contextos existenciales (Ibáñez, 2003), no sólo inciden el discurso identitario producido por los niños, niñas y adolescentes (Marc y Picard, 1992), sino que también contribuyen a la producción de subjetividades por cuanto significan normas, valores, lenguajes, herramientas, procedimientos y modos de hacer (Castoriadis, 1988). En relación al objetivo de la integración cívica y social, estos niños son protegidos a través de un proceso dirigido a la reproducción del magma de significaciones imaginarias (Castoriadis, 1988) de la protección. Dicho magma descansa en una imagen negativa de los niños/as institucionalizados, guiando el modo de hacer cotidiano hacia el control social y estableciendo normas, límites y rutinas.

La representación negativa de la infancia en acogimiento residencial se debe en gran parte a la idea de normalización, activada con la reforma de 
1987, que identifica a la familia (adaptada socialmente) como la institución privilegiada para la integración y el desarrollo del niño y la preservación de los valores sociales. De este modo, en el imaginario institucional, mientras que el acogimiento familiar y/o la adopción son vistas como formas «normalizadas» de protección, existe una tendencia a identificar el acogimiento residencial con recursos dirigidos a niños/as y adolescentes que por diversas razones no pueden crecer en una familia, lo que conlleva un daño irreversible y/o genera conductas antisociales.

Horarios, normas y rutinas

Consecuencia del marco institucional y simbólico de la protección, las identidades individuales y sociales de estos niños/as y adolescentes se estructuran en torno a la idea de normalización, donde la normalidad significa la interiorización de las normas y rutinas y la idea de familia es fundamental en la producción de sus subjetividades.

De este modo, algunos niños pusieron de manifiesto la importancia de sus comportamientos dentro y fuera de los recursos de acogimiento, las normas que tenían que cumplir y cómo estas prácticas dan forma a su identidad actual y futura:

En esta casa cuando entre tenia mis manias, defectos... pero poco a poco, he ido mejorando, como persona (creo yo). Me han enseñado cosas, educado [...] para que cuando salgamos de esta casa seamos gente bien: educados generosos y buenas personas (Carta).

De hecho, los horarios se constituyen como una parte central en los relatos producidos por los niños y niñas sobre la vida cotidiana en los centros, residencias u hogares y la incidencia de éstos en la imagen de sí mismos:

Gracias a los horarios aprendes a ser un poco más organizada (19).

Incluso, narran su historia de vida como una secuencia de fechas, situando los momentos vividos como estructuradores de sus biografías en torno a la edad y el ingreso en las instituciones de protección:

Ahí estuve casi un mes o dos meses antes de cumplir [...] 8 meses antes de cumplir los 13, que eran los 12, y hasta los 16, hasta un mes antes de cumplir $\operatorname{los} 15$ (I2).

Hola me llamo Pedro, tengo 13 años casi 14 años porque el 24 de diciembre los cumplo. En la casa estoy muy bien siempre se han preocupado por mi llevo aquí 7 años (Carta). 


\section{Familia como institución generadora de normalidad}

Para la mayoría de los niños, niñas y jóvenes entrevistados, la idea de normalidad corresponde con el hecho de vivir y crecer en una familia; lo cual genera cierta ambivalencia identitaria. Algunos jóvenes sienten que han tenido una vida e infancia normales porque, aunque no hayan vivido con sus familias, han crecido en un ambiente normalizado (residencia infantil). Sin embargo, ellos/as sienten que el hecho de no haber tenido una familia los diferencia de otros niños.

No me veo diferente, bueno, he tenido una vida diferente a la gente normal que han vivido con sus padres pero me veo normal, como cualquier chica (I5).

La idea de familia también funciona como referente para designar la normalidad, de la misma manera que es la legitimación de las normas por parte de los/as jóvenes.

Con mi educador-tutor muy bien, muy bien. Es como mi padre [...] Para mí la residencia de menores es como tu familia [...] con horarios, todo estructurado pero una vida normal que no te influye luego, al salir... que vamos, que eres como otra persona normal (I3)

Para mí eran mi familia ¿sabes? (17).

En estas narrativas, la idea de familia funciona como factor de legitimidad de las normas impuestas o como fundamento de las «buenas relaciones», coincidiendo con una experiencia de ruptura respecto a la familia de origen (tutelas). Por el contrario, aquellos que estuvieron en guarda y sostuvieron la relación con sus familias invierten el discurso. Es decir, la no aceptación de la norma deviene de la no identificación de la misma con la figura familiar.

Yo no voy a estar un mes en una habitación y, además, vosotros podéis ser mis tutores pero no sois ni mi madre, ni mi padre para decir con quién me tengo que juntar, si tengo que ir a fútbol o no tengo que ir (I2).

Es decir, los niños/as y jóvenes no sólo construyen sus identidades reelaborando el marco normativo e institucional, sino que éste también es reinterpretado para la aceptación, oposición y/o negociación de las prácticas cotidianas ejercidas en los recursos de protección. De esta manera, los niños y niñas en acogimiento residencial muestran su capacidad de contestación ante el sistema instituido que, lejos de ser interpretado como parte de una conflictividad atribuía, manifiesta las distintas formas de ejercer su agencia como sujetos sociales e individuales. 
Pertenencias y categorías sociales en el magma simbólico de la protección

Las identidades se construyen sobre categorías de referencia y comparación, componiendo pertenencias que integran al grupo, pero también implican la identificación personal, el establecimiento de vínculos afectivos, la asunción de normas y hábitos compartidos y el sentimiento de solidaridad hacia los otros miembros del grupo (Weil, 1996). Consecuencia del magma simbólico $y$ de significaciones de la protección, en las narrativas de los niños, niñas y jóvenes se encuentran constantes referencias a las categorías ser niño/a y ser un niño/a de centro.

\section{Ser niño/a, ser adulto}

Para estos niños/as y jóvenes, la edad se corresponde -en relación proporcional- con la consideración percibida por parte de los adultos y las instituciones. De hecho, es a partir de las diferencias conceptuales en la identidad entre niños/as y adultos (Jenks, 1996) que surge el problema mismo de la situación de los niños y niñas como ciudadanos (James, 2011: 161), pues la ciudadanía plena se asocia a la adultez en las sociedades democráticas occidentales.

Así, en las narrativas producidas por los jóvenes es fácil encontrar referencias a la dicotomía niño/a-adulto pues ser adulto significa culturalmente ser responsable, independientemente de la edad biológica. Llegar a ser adulto es identificado con tener un «buen» comportamiento en oposición al de ser niño/a (o adolescente). El niño/a es percibido como un ser de personalidad inherentemente problemática o conflictiva. Ser adulto no se relaciona necesariamente con la edad, si no con la adquisición de ciertos tipos de comportamiento caracterizados por el control de las emociones y la tranquilidad personal:

Yo era problemático en casa, como un niño de 12 o 13 años [...] fui por si también quería yo no estar tan alborotado como estaba y hacerme adulto con 12 (I2).

Por otra parte, el incremento de la edad es asociado por los niños/as y jóvenes con ser tratados como personas independientes y con tener mayor libertad para actuar. Esto es visto como consecuencia de la autoconsciencia sobre las cosas y lo que ocurre alrededor, en la medida en que se acercan a la adultez. Los jóvenes asocian el estatus de poder con la edad, fruto de todo un discurso normativo aprendido y de carácter paternalista:

Un día, por ejemplo, nos acostábamos más tarde, pero porque la monja nos dejaba, veía que podíamos hacerlo porque éramos un poco mayores y responsables (I5). 
La adquisición progresiva de autonomía es asociada a la madurez y ésta con la edad, pero también con las experiencias vividas. El mundo de la infancia es el mundo del juego y éste el de la no consciencia de lo que ocurre alrededor.

A mí me ha hecho una persona muy madura [...] Me lo ha dicho mucha gente que ninguna chica de 20 años es tan madura (I5).

Los educadores para dejarme salir me exigían madurar, que madurara, que fuese más responsable y que no fuese una cabra loca (I7).

La madurez es asociada a la independencia (no interdependencia) o ausencia de ayuda en la toma de decisiones, fruto del modelo liberal de ciudadanía instituido que descansa en un sentido privado y negativo de la libertad y de la autonomía, característico del modelo liberal de ciudadanía imperante.

\section{Ser un niñola de centro}

Esta pertenencia social del niño/a implica su inclusión en la colectividad de la categoría o grupo niño/a de centro:

Soy una niña tutelada en un piso de protección (Carta).

Sin embargo, la pertenencia al grupo niño/a de centro no es experimentada necesariamente en base al sentimiento de lealtad hacia ella. La inclusión, más bien, se realiza mediante la asunción del rol niño tutelado dentro de la colectividad y de la apropiación e interiorización de, al menos, parte del marco simbólico-cultural instituido por el sistema de protección, es decir, de la idea de familia, la importancia de las normas en la producción de subjetividad y la representación del niño/a protegido/a como un ser conflictivo y culpable.

El centro terapéutico es el peor centro que te puedas echar a la cara porque va la peor gente. En el mío no iba gente mala, iba gente... vamos, no hay gente mala nunca pero sí hay niños traviesos, no malos (I7).

En el centro, el grupo de chicos que estábamos era como nuestra familia, más o menos teníamos algo en común (I1).

El ser un niño/a de centro funciona como etiqueta y significado en la constitución de su propia subjetividad. Vivir en un recurso de acogimiento residencial contribuye al sentido de sí mismos y de su identidad.

Soy una niña que está en un piso de protección. Desde que llegué aquí me alegre mucho. Al principio creí que era un internado pero me he dado cuenta que no tiene que ver nada con un internado. Estoy en una casa normal (Carta).

Frente a la idea colectiva de la infancia protegida como un grupo relativamente homogéneo donde los niños comparten características y experiencias similares, los niños/as y adolescentes protegidos establecen distinciones internas 
o subcategorías en la protección y, en función de ello, atribuyen determinadas características a los diferentes recursos de acogida.

En todos los discursos, las residencias de primera acogida ${ }^{5}$ son vistas como unos centros muy negativos e invasores y son identificados como recursos dirigidos a los niños/as inmigrantes no acompañados y estos, a su vez, con el conflicto social.

Ir la residencia esta de primera acogida... porque había allí gente muy conflictiva (I3).

Me mandaron a residencia de primera acogida y se notaba que [...] había muchísima gente que sobre todo eran extranjeros, chicos marroquís que vienen sin papeles y tal... y yo eso... ya no me meto ahí (19).

Los niños/as y adolescentes protegidos responden al marco político- normativo cultural y moral compartido socialmente. En este escenario el conflicto social es identificado con los «otros» (niños/as inmigrantes no acompañados). Pese a que estos jóvenes han vivido parte de su infancia o adolescencia sin sus familias, al igual que los jóvenes migrantes, su experiencia de separación familiar es vivida como algo involuntario; mientras que la experiencia de los «otros» es vista como algo decidido por ellos y, por tanto no normal.

\section{Los procesos de construcción ciudadana}

Como ya pusieran de manifiesto estudios como los de R. Thomson et al. (2004), los jóvenes entrevistados tienden a identificar la ciudadanía con la edad adulta. Esta asociación responde al modelo clásico de ciudadanía en el que el ciudadano por antonomasia es el adulto independiente económicamente y al que no sólo se le atribuye la ciudadanía plena, sino también las características de madurez, responsabilidad y autonomía; por lo que se le permite adquirir plenos derechos y participar política y socialmente.

Para los niños/a y jóvenes, cumplir 18 años supone la adquisición de la adultez pero, sobre todo, la oportunidad real de hacer libremente, tomando y poniendo en práctica sus propias decisiones. Hecho que ha ser visto como una consecuencia directa del impacto de las políticas de la infancia sobre la vida de los niños y niñas. Es más, para los niños/as y jóvenes protegidos, la ciudadanía es identificada sobre todo con la posibilidad de la (auto)determinación, en contraste con una experiencia vital marcada por la exclusión tanto del proceso y organización de su propia protección como de las prácticas establecidas

5. Macrocentros de tránsito donde los niños /as y adolescentes permanecen hasta que la administración autonómica les asigna otro recurso de acogimiento residencial. 
para sus vidas cotidianas; debido a su condición de «menores de edad»y, sobre todo, como «niños o niñas de centro», vulnerables o conflictivos.

Yo tenía ganas de cumplir 18, de hacerme mayor [...] sólo quería tener 18 años y ser adulta (I5).

Incluso en los casos en los que los jóvenes entrevistados tienen un diagnóstico de enfermedad mental, sus elecciones se dirigen hacia la ansiada independencia y autonomía.

Aquí me dan una ayuda al mes que es de 720 euros y por el piso pago 270 euros y me incluye la luz, el agua y todo [...] De los 270 que yo pago me dan 41,60 a la semana para ir a hacer la compra. Yo me voy con la moto al supermercado y cojo y digo 'Hoy quiero patatas, como es viernes, me voy a comprar una bolsa de patatas' y cocino y eso. Y se está aquí mejor que en España (I1).

El establecimiento de las propias reglas (cotidianas), como ir a hacer la compra o decidir qué comer y cuándo, es visto como un signo inequívoco de autonomía, pero sobre todo como el margen de libertad (social) a partir del cual se les permite ser tratados como personas, independientemente de su edad o (in) capacidad.

Al igual que algunos investigadores han puesto de manifiesto (Lister et al. 2003) en la experiencia del Reino Unido, los jóvenes entrevistados representan la idea de ciudadanía a partir de dos modelos fundamentalmente: 1) independencia económica; y 2) participación social, ambos relacionados con el proceso de inclusión/exclusión socio-política y social. No obstante, en las narrativas producidas $-\mathrm{y}$ en el caso español- se puede encontrar un tercer modelo de la construcción de la ciudadanía asociada a la familia.

\section{Ciudadanía como independencia económica}

Acorde con el modelo de independencia económica, los jóvenes consideran el empleo como la expresión de la adultez y el estatus ciudadano, en la medida en que obtienen un salario y, con ello, la independencia económica de las instituciones. Para ellos, disponer de ese salario (aunque sea bajo y precario) les legitima para tomar sus propias decisiones y ordenar sus vidas.

Y nada, yo ahora que me ha metido mi vieja en un piso de mayores, debuty, la verdad y me estoy sacando la ESO [... ] Mi hermana me va a meter a currar en Springfield (I4).

Yo llevo ya cuatro años trabajando y, bueno, yo antes era una niña y tú me podías decir 'déjame dinero' y te lo dejaba. Claro, yo no sabía pero lo he aprendido... y el tema de no gastar tanto o si gasto apuntarlo (I5). 
No podías tomar muchas decisiones, tampoco era mucha autonomía [...] Yo porque empecé a trabajar [...] Ellos decían que yo a las 21 h tenía que estar en la residencia y yo decía que no. Ellos decían que las normas eran para todos y yo 'ya, pero es que todos no están trabajando' (17).

Cabe señalar que estos niños y niñas, en muchas ocasiones, se ven forzados a incluirse en el mercado laboral al cumplir los 18 años porque no disponen de la protección y soporte económico de sus familias y/o las instituciones que hasta la fecha les habían «protegido». Por tanto, ellos entienden que la independencia económica es una forma de emancipación de la autoridad del sistema de protección pero también, para muchos de ellos, la única manera de sobrevivir.

Cuando hice los 18, dijo el director 'Ya tienes 18, ya te puedes ir' y no había ni un piso, no tenía arreglados los papeles para ir a ningún curso, ni dónde te vas a ir... Eso era: puertas abiertas, sal y búscate la vida (I1).

La infancia es a menudo considerada como un periodo de especial vulnerabilidad y pasividad durante la cual los niños/as se preparan para su vida futura como adultos. La noción del «devenir» constituye una visión romántica de la infancia caracterizada por la ausencia de preocupaciones, proponiendo el énfasis en la preparación de los niños y niñas para la vida adulta a través de un conjunto de prácticas supervisadas y controladas que incluyen la educación formal.

Reflejo de esta imagen colectiva, en las narrativas producidas por los jóvenes, la idea de responsabilidad y la adquisición de capacidades adultas, marcadas por la noción de la independencia (económica), se relaciona con la participación en la educación formal.

La imagen de la educación formal es identificada, por un lado, como una experiencia que provee de madurez y culmina en la adquisición de la condición de persona y por otro, como un vehículo para cambiar activamente identidades futuras y para alcanzar un estatus social -y económico- más alto.

Eso de limpiar las piscinas... en vez de los cursos de matemáticas que teníamos sólo en invierno, ahí aprendíamos más. Y yo porque tenía 17 pero el Ramón, el que tenía 14, no se podía formar bien y la consecuencia habrá sido que ahora no habrá sido nada (I1).

Cuando entré en el piso, en el instituto me iba mal porque tenía una situación familiar difícil y a mí el instituto no me parecía... [...] Lo que pasa es que pues era un ignorante, era un chaval (I8).

Porque por ahora el estudio es mi vida, quiero conseguirlo (Carta). 


\section{Ciudadanía como participación social}

El modelo de participación social se relaciona con la idea de ciudadanía social. Muchos de los jóvenes entrevistados mostraron actitudes proactivas hacia la ayuda, expresando su interés por actividades de voluntariado o por realizar estudios relacionados con la «ayuda».

Yo siempre he tenido una vocación para ayudar a las personas. Siempre he sido muy de ayudar a la gente, me ha gustado ayudar a la gente y había pensado ser auxiliar de clínica veterinaria [...] o auxiliar de enfermería (I9).

No obstante, los jóvenes no consideraron este tipo de actividades desde una perspectiva filantrópica, sino más bien como una forma de desarrollar prácticas y proveer de oportunidades a otros niños/as para que puedan ejercer sus derechos.
Yo le he hablado con mis amigos y les digo 'me gustaría trabajar con niños' y me dicen 'a ver, piensa tío, te ha pasado a ti esto...' y digo 'pero es que me gus- taría, es que me encantan los niños' 'ipero te verías como para ponerles leyes o para ponerle la mano a un niño?' 'no, no, que va de educador no, de jardín de infancia en una guardería...' Es que siempre me han gustado los niños. Yo siempre les digo a mis amigos que si me hago educador, tengan por seguro que yo sería educador tirando a amigo [...] hay educadores y educadores. Hay educadores que si tienes un problema puedes hablar con ellos (I2).

Como muestran los extractos anteriores la ayuda se relaciona con la condición de persona y ésta con el reconocimiento del «otro-niño/a»o «ponerse en la piel de» como arguye la siguiente interlocutora:

A mí me encantaría ser educadora porque no es lo mismo ser educador que ser un niño ¿sabes? Estar en la piel de un niño, no es lo mismo. Y si yo en algún caso, pudiera estudiar algo, estudiaría eso porque yo me he puesto en la piel de los niños (17).

Por otra parte, los jóvenes manifestaron no haberse sentido reconocidos como personas de pleno derecho durante su experiencia de protección, lo que es visto por ellos como una forma manifiesta de exclusión social. Por tanto, para ellos, la participación social significa también presencia socio-política en los espacios públicos, la cual es fundamental para ser considerado ciudadano (en un sentido sustantivo).

\section{Ciudadanía como familia}

Fruto de una tradición socio-cultural e histórica fundamentada en la institución familiar como espacio privilegiado para la formación e inclusión cívica, la construcción familiar de la ciudadanía se constituye como el tercer modelo de ciudadanía representado en los discursos delos niños/as y adolescentes. 
Incluso para algunos de ellos formar una familia implica necesariamente tener hijos. La familia es considerada como el entorno donde los niños y niñas tienen la oportunidad real de ser tratados y considerados como «personas» desde el presente y en el futuro.

Mis planes: sacarme los estudios y luego formar una familia, aparte de que me gustan mucho los niños, formar con mi novia una familia. Me gustaría hacer bachillerato y luego trabajar de mozo de almacén o ya que estoy tan metido en el tema de niños, trabajar en el tema de niños (I2).

Como señala Gil Calvo (1988), en nuestra sociedad para que se dé la integración social (y cívica) completa no sólo hay que disponer de un empleo remunerado estable y propiedades (independencia económica) o ejercer alguna clase de participación pública (voto, opinión, etc.), en el imaginario colectivo, hay que contraer responsabilidades familiares (conyugales, paternales y domiciliares).

\section{Conclusiones}

El discurso colectivo de los niños/as y jóvenes institucionalizados es bastante homogéneo. No obstante, se advierte una mayor incidencia de la variabilidad «régimen de protección» (guarda o tutela) como determinante de la producción de subjetividades.

Las narrativas de los niños/as y jóvenes entrevistados ponen de manifiesto su capacidad de acción y contestación ante la imagen socialmente instituida del niño institucionalizado, aunque al mismo tiempo, el análisis del material empírico muestra las tensiones existentes en los relatos de los niños entrevistados sobre sus identidades y pertenencias. Esta dualidad debe ser vista como fruto del impacto del marco simbólico de la protección; así como el producto, aunque reelaborado, del conjunto de prácticas cotidianas en los recursos de acogimiento residencial. Por un lado, los niños, niñas y jóvenes tienden a legitimar su exclusión ordinaria de la toma de decisiones según la edad y a la (in)capacidad atribuida, particularmente aquellos que estuvieron acogidos en régimen de tutela. Por otro, cada uno de sus relatos contiene el deseo o la frustración de no haber sido tenidos en cuenta en los asuntos que afectan a sus vidas, lo que queda referido en la forma en la que construyen y proyectan su ciudadanía.

De la misma manera, se muestra cómo el contexto simbólico de la protección incide en la visión de estos niños/as y adolescentes sobre los significados del ser adulto y ser ciudadano, identificándose tres modelos de ciudadanía dominantes: independencia económica, participación social y formación de la familia. Mientras que los dos primeros modelos responden a una concepción 
de la ciudadanía característica del modelo clásico liberal, la tercera incorpora los valores culturales específicos del caso español, atribuyendo especial relevancia a la formación de una familia en la adquisición de la ciudadanía plena.

\section{Bibliografía}

BERGER, P. y LuCKMAnN, T. (1967). The Social Construction of Reality. New York: Doubleday.

BOLETÍN DE DATOS ESTADÍSTICO DE MEDIDAS DE PROTECCIÓN A LA INFANCIA. (datos 2013), n. ${ }^{\circ} 16$. Recuperado de http://www.observatoriodelainfancia.msssi.gob.es/productos/home.htm

CASTORIADis, C. (1988). Los dominios del hombre: la encrucijada del laberinto. Barcelona: Gedisa.

COCKBURN, T. (1998). Children and Citizenship in Britain: A Case for a Socially Interdependent Model of Citizenship. Childhood, 5(1), 99-117.

CoHEN, E. (2005). Neither Seen Nor Heard: Children's Citizenship in Contemporary Democracies. Citizenship Studies, 9(2), 221-240.

GAITÁN, Lourdes. (2014). De «menores» a protagonistas. Los derechos de los niños en el trabajo social. Madrid: Consejo General de Trabajo Social.

GIL CALVO, E. (1988). «La discontinuidad social y la integración de los marginados». Revista Alfoz, 57, 45-54.

Goffman, E. (1961). Asylums: Essays on the Social Situation of Mental Patients and Other Inmates. New York: Dubledzay.

IBÁÑNEZ, J. [1979] (2003). Más allá de la Sociología. El grupo de discusión: Técnica y crítica. Madrid: Siglo XXI.

INFORMES, ESTUDIOS E INVESTIGACIÓN. (2012). Estándares de calidad en acogimiento residencial- EQUAR. Ministerio de Sanidad, Servicios Sociales e Igualdad. Recuperado de: http://www.observatoriodelainfancia.msssi.gob.es/ productos/home.htm

JAMES, A. (2011). To be (come) or not to be (come). Understanding children's citizenship. The ANNALS of the American Academy of Political and Social Science, 633(1), 167-179.

JENKS, C. (1996). Childhood. London: Routledge.

LIEBEL, M. (2008). Citizenship from below: children's rights and social movements. En J. Williams y A. Invernizzi (eds.), Children and Citizenship (pp. 32-43). London: Sage.

Lister, R., SMith, N., Middlenton, S. y Cox, L. (2003). Young People Talk about Citizenship: Empirical Perspectives on Theoretical and Political Debates. Citizenship Studies, 7(2), 235-253.

LISTER, R. (2007). Why citizenship: Where, when and how children?. Theoretical Inquiries in Law, 8(2), 693-718. 
MARC, E., y PICARD, D. (1992). La interacción social: cultura, instituciones y comunicación. Barcelona: Paidós.

Mead, G.H. (1934). Mind, Self and Society. Chicago University Press.

Millham, S., Bullock, R., y CherRet, P. (1975). A conceptual scheme for the comparative analysis of residential institutions. En J. Tizard, I. Sinclair y R.V.G. Clarke (eds.), Varieties in Residential Experiences (pp. 203-224). London and Boston: Routledge \& Kegan Paul.

Moosa-Mitha, M. (2005). A Difference-Centred Alternative to Theorizing of Children's Rights. Citizenship Studies, 9(4), 369-388.

Moss, P. (1975). Residential Care of Children: A General View. En J. Tizard, I. Sinclair y R.V.G. Clarke (eds.), Varieties of Residential Experiences (pp. 17-51). New York: Routledge \& K. Paul.

RAMiro, J. (2015). De los derechos para la infancia a la ciudadanía de los niños. Estrategias de orden y control social (Siglos XVIII al XXI). e-SLegal History Review, 19, 1-42.

Roche, J. (1999). Children: Rights, Participation and Citizenship. Childhood. $6(4), 475-493$.

Thomson, R., holland, J., McGrellis, Bell, R., Henderson, S. y Sharpe, S. (2004). Inventing Adulthoods; A Biographical Approach to Understanding Youth Citizenship. Sociological Review. 52(2), 139-294.

TizARD, J., SinClair, I., y ClARKE, R.V.G. (1975). Varieties of Residential Experiences. New York: Routledge \& K. Paul.

WeIL, S. [1943] (1996). Echar Raíces. Madrid: Trotta. 